\title{
Sheehan Syndrome
}

National Cancer Institute

\section{Source}

National Cancer Institute. Sheehan Syndrome. NCI Thesaurus. Code C35300.

An uncommon cause of hypopituitarism seen after severe postpartum hemorrhaging.

Prolonged hypovolemia leads to ischemic necrosis of the pituitary. Clinical signs typically present in the puerperium and include failure to begin lactation, fatigue, hypotension and eventual amenorrhea. Clinical course is usually mild, however extreme cases may progress to adrenal failure. Prognosis is most favorable when hormone replacement is initiated soon after symptom onset. 